\title{
Innovations
}

\section{Implementation of pediatrics residency accredited program in Singapore}

\section{Authors}

Chong Chia Yin 1,2,3*; Serene A.K. Ong4; Yeo Ai Ling4; Chay Oh Moh',2,3

\section{Abstract}

In 2009, Singapore began replacing the British-based specialist training that constituted its postgraduate medical education with a United States-based Residency program, which is more structured. Singapore is the first country outside the United States to be accredited by the Accreditation Council for Graduate Medical Education - International (ACGME-I). This paper reviews the recent changes in the context of the Pediatrics program at the KK Women's and Children's Hospital, one of the five specialties chosen to launch the new residency programs. Most of the changes were made for accreditation purposes, so as to align with the requirements set by ACGME-I; however, local content and Ministry of Health (MOH) stipulations were also taken into account when designing the new pedagogical and training curriculum. Areas discussed include faculty numbers and development, concurrent training of trainees from the previous system, selection of residents, resident training, resident duty hours, mentors, curriculum, rotations, continuity clinics, scholarly activity, postgraduate exams, remediation, and chief residents.

\section{Keywords}

residency; postgraduate medical education; ACGME-I; international accreditation; curriculum development

\section{Introduction}

Since the 1960s, British-based specialist training has been the system of choice for postgraduate medical training in Singapore, consisting of two phases: the Basic Specialist Training (BST) and Advanced Specialist Training (AST). The challenges faced with the British-based system are that it was time-based and lacked a formal training program, with a large proportion of trainees going through rotations just to fulfill the bond requirements imposed for entering medical school. However, in 2009, the Ministry of Health $(\mathrm{MOH})$ chose to replace the old system with a United States-based residency program. The new system consists of a more structured training program that is competency-based. ${ }^{1,2}$

This paper reviews the recent changes in postgraduate medical education in Singapore within
'KK Women's and Children's Hospital, Singapore

${ }^{2}$ National University of Singapore (NUS) Yong Loo Lin School of Medicine, Singapore

${ }^{3}$ Duke-NUS Graduate Medical School, Singapore.

${ }^{4}$ Academy, Group Education, Singapore Health Services Pte. Ltd, Singapore.

* E-mail: Chong.Chia.Yin@kkh.com.sg.

\section{Cite this article as:}

Yin CC, Ong SAK, Ling YA, Moh Co. Implementation od pediatrics residency accredited program in Singapore. Innovations in Global Health Professions Education. 2015:4. http://dx.doi.org/10.20421/ighpe2015.4

This is an open access article distributed under the terms of the Creative Commons Attribution license CC BY 4.0, which permits unrestricted use, distribution and reproduction in any medium, provided the original work is properly cited. 
the context of the pediatrics program at the KK Women's and Children's Hospital (KKH), Singapore's only integrated hospital for women and children, and one of the largest children's hospital in the region.

\section{Changes in Postgraduate Pediatrics Training}

Previously, postgraduate training was handled by Ministry of Health Holdings (MOHH), but now the administration is largely passed to the Sponsoring Institutions (SI). A Designated Institutional Officer (DIO), Associate Designated Institutional Officer (ADIO), together with Program Directors (PD), Program Coordinators (PC), and faculty were appointed to facilitate the coordination of the residency programs.

A variety of changes were effected with the switch in systems so as to meet the accreditation requirements by the Accreditation Council for Graduate Medical Education - International (ACGME-I). Areas affected include: faculty numbers and development, concurrent training of BST trainees, selection of residents, resident training, resident duty hours, mentors, curriculum, rotations, continuity clinics, scholarly activity, postgraduate exams, remediation, and chief residents.

\section{Faculty numbers and development}

Additional manpower was necessary for two main reasons: for faculty to have protected time for education, and to meet the ACGME-I requirements for faculty to resident ratio. The residency program requires a 1:1 faculty to resident ratio with a 1:6 core faculty to resident ratio, and the current faculty had to be trained to understand and evaluate residents on the competencies of the program. This exacerbated the manpower shortage that resulted from loss of specialists to the private sector. With funding subventions from $\mathrm{MOH}$, specialist manpower was recruited from overseas. At the same time, so-called nurse extenders were appointed from experienced nurses to help with the repetitive service work with only limited educational value.

Faculty attended development talks to receive ongoing training; topics included the six core competencies and how to teach them, providing feedback, mentoring, managing, and evaluating residents.

\section{Concurrent training of BST trainees}

There were 26 BST trainees remaining from the old British-based system when the residency program was launched. In order to transition the existing BST trainees into the residency program, some aspects of the residency program were incorporated into the old system, creating a hybrid system.

The main focus of the old system was on medical knowledge, patient care, and communication skills. The residency program has an additional three new competencies: professionalism, problem-based learning and improvement, and system-based practice. In addition, a variety of other changes were incorporated into the old system, such as, In-Training Exams, end of monthly rotation evaluations, protected training time, core didactic lectures to match the new residency requirements, mini-clinical evaluations (miniCEX), case-based discussions (CBD), selfreflective entry, and limits on 80 duty hours per week. These changes were complementary to the remaining requirements of the old system.

However, remnants of the old system were retained for the BST trainees, including six-month rotations in Neonatology and Children's Emergency, instead of three months for residents, rotation supervisors instead of longitudinal mentors, and no restriction on new patient care for post-night duty. Unlike residents, BST trainees did not have to log onto ACGME-I for case diagnoses or procedure logs, or log in duty hours; instead they retained hard copy logs. The BST trainees also had different duty hours and leave restrictions from the residents.

\section{Selection of residents}

Two cohorts form the pool of applicants for residency, namely PGY1 (post-graduate year 1) and PGY2 and beyond. PGY1 were new graduates from the National University of Singapore (NUS) and Duke-NUS, who had just finished their undergraduate training, while PGY2 and beyond were individuals who had completed internship, as well. However, in our program, all candidates were expected to enter residency training at Residency Year 1 (RT), 
regardless of prior experience. We did not fast-track residents with prior experience because there were no eligible residents who had completed the required paperwork, such as mini-CEX, CBD, procedure and case logs.

In the first year, applicants underwent an $\mathrm{MOHH}$ interview, which was conducted from November to December each year. The interview panel comprised the Residency Advisory Committee (RAC) chairman, and a representative each from Singhealth Services (SHS) and the National University Health Services (NUHS), the only two Sls that run pediatric residency programs in Singapore. This interview was intended to identify candidates that are suitable for residency training.

From the second year onwards, interviews, known as Multiple-Mini Interviews or MMls, were conducted to determine suitability for a specific specialty. The SIs also organized annual open houses to help applicants gain a better understanding of each institution and what it offers. Once applicants had selected their institution and programs had selected their applicants, a computerized system matched an applicant to a program at one of the institutions.

Thirteen residents were matched in our institution's first intake in May 2010, and 16 residents were matched in May 2011, and 15 in May 2012. In all three years, about half were from PGY1 and half from PGY2 and beyond. Although we had targeted to take in 16 candidates in the 3rd year, one candidate did not clear his undergraduate exams and could not be selected.

\section{Resident training}

New residents were educated on the six core competencies and how the evaluation takes place. The evaluative tools were based on a nine-point Likert scale, which was completely new to the program. Evaluations of residents were completed every six months for the records of $\mathrm{MOHH}$, and yearly for the residents' year-end bonuses. In addition, ACGME-I also mandated a yearly evaluation to be entered into the accreditation data system, which is an Internet-based data collecting system. However, the MOHH and ACGME-I evaluations were not identical with 21 and 29 criteria respectively; in addition, ACGME-I mandated that annual evaluations be mapped to the stages of novice, advanced beginner, competent, and expert.

\section{Resident duty hours}

The Residency program imposed a restriction of no more than 80 duty hours per week. Trainees under the BST system averaged 60-75-hour work weeks, so keeping to the restriction was not an issue. However, this proved difficult for PGY1 residents when they were rotated to General Surgery and Internal Medicine at Singapore General Hospital (SGH) or Changi General Hospital (CGH). To help ease the problem, SHS began monitoring duty hours on a weekly basis so that residents who exceeded the 80hour work week could be compensated the following week. As a result, excessive duty hours did not surface as often.

In addition to the 80-hour work week, residents were also required to fulfill a minimum of 16 hours protected training time (PTT) per month. Orientation training, bedside teaching, core didactic lectures, simulation training, supervised clinics, and procedural skills training were protected, thereby enabling residents to fulfill this requirement.

\section{Mentors}

Under the previous system, supervisors were assigned to trainees for the duration of a rotation. Now, mentors are longitudinal, following residents throughout their program, and are assigned based on residents' requests for gender or subspecialty. In 2010, a half-day faculty development workshop was organized to train faculty members to become good mentors. Since mentors were not supposed to evaluate their resident mentees during any rotation, another faculty member who was not mentoring that resident had to be assigned for the monthly rotation evaluations. Resident mentees were required to meet their mentors at the start of residency and subsequently quarterly. These meetings had to be documented and residents were also required to complete an individualized learning plan, which was updated yearly.

\section{Curriculum}

One challenge for the medically trained PD was developing the new pedagogical curriculum. The 
majority of the current curriculum was developed from other residency programs in the United States, and modifications were made to meet the requirements during rotations. For example, the program's curriculums for rotations like Neonatology, Children's Intensive Care, and Children's Emergency had to be modified to show progressive capability and responsibility. The curriculum subcommittee met regularly each year to discuss and update each subspecialty's curriculum.

\section{Rotations}

Due to the different graduating schedules of the two undergraduate medical schools in Singapore, rotations in pediatrics were different for PGY1 (NUS, and Duke-NUS) and PGY2 (graduates who completed internships). Moreover, the staggered rotation schedules also meant that we were unable to accept international medical graduates who started their internship in different months from NUS or Duke.

Since NUS graduates started internship in May, two months earlier than Duke-NUS applicants who started in July, NUS graduates underwent fourmonth rotations each in Internal Medicine (IM) and General Surgery (GS) in residency year 1 (R1), while the Duke-NUS residents underwent three months each of rotations in IM and GS. This enabled both PGY1 cohorts to be on par for the completion of 36 months of pediatrics-related rotations. However, the $\mathrm{IM}$ and GS rotations were not recognized as part of the pediatrics residency, therefore PGY1 graduated six to eight months later than PGY2 and beyond.

Table $1^{3}$ states the Residency rotations for R1, R2, and R3 and also gives the rotations for Senior Residency. Elective rotations were included for R2 and R3; it could be spent by weeks or a whole month at pediatric otolaryngology, pediatric ophthalmology, pediatric orthopedics, pediatric anesthesia, diagnostic imaging, child psychiatry, or palliative medicine. Overseas elective rotations were also available for residents to rotate to Duke Durham, United States or the Imperial College, London, United Kingdom. Senior residency was mandated as the RAC still required trainees to complete an additional three to four years of general pediatrics or subspecialty before they qualified as a specialist pediatrician. This was similar to the United Kingdombased system, which also needed an additional three years of Advanced Specialty Training on top of three years of Basic Specialty Training.

\section{Continuity clinics}

Although continuity clinics (CCs) were not a mandatory requirement by ACGME-I, it was implemented in our program as we saw the value of this experience for all residents. The focus of CCs was anticipatory and preventive care. CCs were blocked for the three months in SGH Neonatology rotation and during the month of rotation in Neonatal or Children's Intensive Care, and Developmental Disorders.

However, CCs came with a variety of issues such as locating clinic space, clashing with subspecialty clinics or subspecialty rounds, and late completion of morning ward rounds, resulting in a late start for morning CCs. To solve these problems, from 2012, all CCs were conducted in the afternoon, and on alternate weeks for each resident, and were only blocked during the elective overseas rotations and when on annual leave.

\section{Scholarly activity}

Residents were required to provide evidence of scholarly activity every six months, according to the RAC stipulated requirements. This included three miniCEX and two CBDs per six months; a reflective entry also had to be submitted every year. Furthermore, residents under our program were also required to provide ten cases or procedure logs per month together with the cumulative minimum number per specific procedure. The problem-based learning and improvement (PBLI) was completed by topic or case representations by the resident at handover rounds, grand rounds, or subspecialty rounds, and by journals critiqued by residents.

\section{Postgraduate exams}

Under the old system, BST trainees needed to pass either of the two exams: Member of the Royal College of Pediatrics and Child Health (MRCPCH), or Master of Medicine (M. Med), Singapore. Under the new system, residents needed to take a further four exams: three annual United States-based, in-training exams (ITE), and the Postgraduate exam (PGE) at R3-4. 
For Residents whoenteredasPGYI

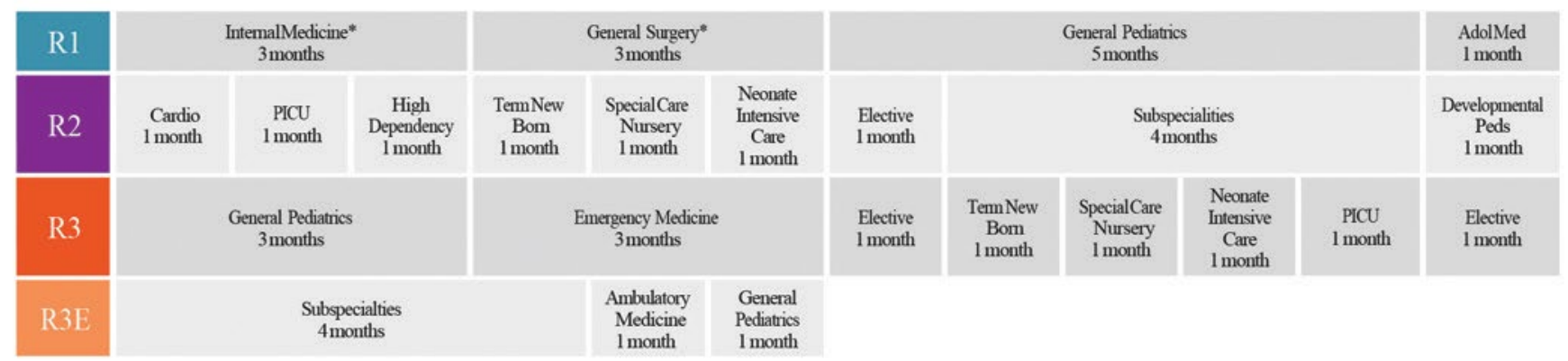

For Residents whoenteredasPGY2 andabove

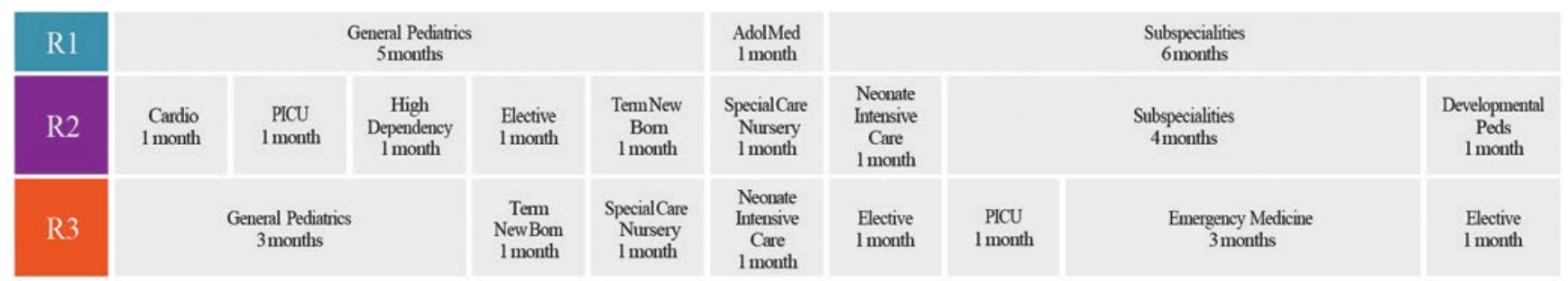

Sample Rotation for Pediatrics Senior Residency

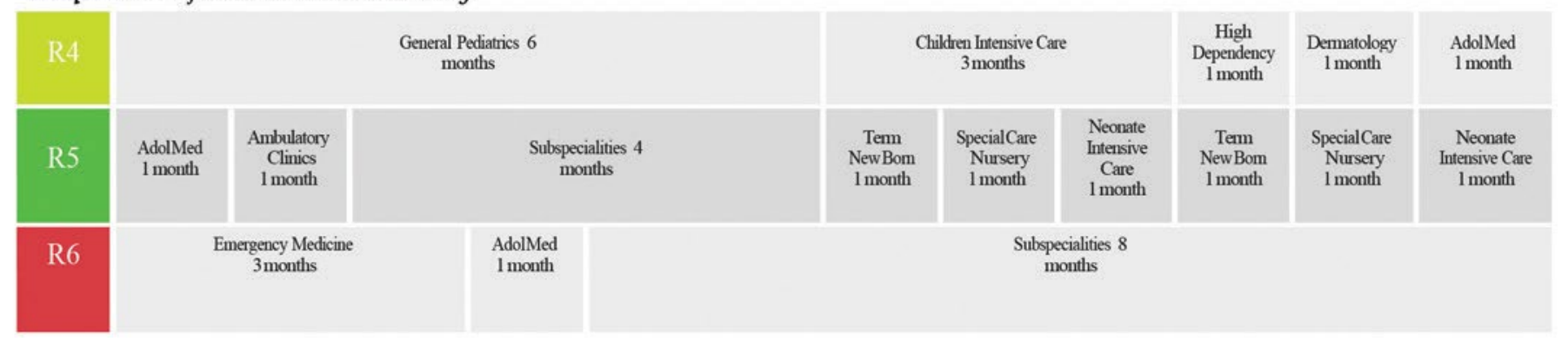

Table 1. Residency Rotations

Previously, in order to graduate, BST trainees only needed to pass the overseas MRCPCH or local conjoint MRCPCH-M. Med exams and attempt the local conjoint MRCPCH-M. Med exams. This was no longer the case for residents; residents must sit for and pass the local conjoint MRCPCH -M. Med clinical exams. Residents must pass this exam to be promoted from year 3 to 4, but they were eligible and could sit for the clinical exam after completing two years of residency. This exam consisted of a theory-based exam of parts $1 \mathrm{~A}, 1 \mathrm{~B}, 2 \mathrm{~A}$, and a clinical exam 2B.

The ITE was a residency requirement and occurred every July; this comprised 200 multiple choice questions and had to be completed within three hours in a computer-based training room with an exam proctor and two invigilators. Unlike some other residency programs in Singapore and in the United States, ITE scores were a promotion criterion for Pediatrics, thus residents had to score the equivalent or better than the national average. In addition, an additional postgraduate theory exam was implemented by $\mathrm{MOH}$ and was slated to start in 2013 for R3 or R4 residents. This PGE exam is a hybrid exam, which includes American Board exam questions for Pediatrics and local questions.

The exams mentioned above were just for Residency. While ACGME-I accredits the 36-months of residency, 
residents in our program were required to complete an additional three years of training in Senior Residency to be eligible for the specialist exit exam. Residents will only be recognized as pediatrician specialists when they pass the specialist exit exam.

\section{Remediation}

In previous academic years, one to three residents from R1-R3 required some form of remediation for the competencies of interpersonal communication skills, professionalism, patient care, or medical knowledge. The remediation process involved obtaining specific information about misbehavior and interviews with a panel comprising the PD, mentor/supervisor, and resident involved. Thus far, all residents who required remediation have been successfully remediated with two pending follow-ups.

\section{Chief residents}

Chief residents, following the United States-style of roles and responsibilities have not been implemented because there have been no graduates from the residency program. However, two AST trainees (who passed the MRCPCH or M.Med exams) who were of registrar grade were appointed as chief residents. Their roles and responsibilities included organizing Monday afternoon protected training time, liaising between residents and faculty, monthly rosters for PGY1 cohort residents, and looking after residents' welfare. Their role did not include planning the 36 months of rotations for residents because this was a formidable task, delegated to one of the associate PDs. These chief residents were given no compensation, other than days off, for their extra administrative duties.

\section{Questionnaire}

We conducted a short questionnaire from August to December 2010 to compare the attitudes of the residents and BST trainees about the new system. The survey had a response rate of $71.8 \%$ (11 residents, 17 BST trainees). Half (53.6\%) agreed that the residency program was more structured and that they received more frequent and effective feedback (64.3\%). Residents were more aware of the six competencies (45.5\%) compared to the trainees (5.9\%), and benefitted from the continuity clinics and better mentorship.
However, only $64.3 \%$ could attend mid-day educational activities most of the time (defined as > $75 \%)$. Reasons for the low attendance included heavy workload (60.7\%), and shortage of manpower (67.8\%).

The BST trainees' attitudes about the residency program were that they felt threatened or shortchanged (41.2\%), angry (17.6\%), bewildered or confused (35.3\%), or neutral $(47.6 \%)$. The majority of BST trainees felt they had to put in more hours to cover for the residents (70.6\%), and that more attention was given to residents and less to the BST trainees (76.5\%).

\section{Conclusion}

Retrospective studies by Lam and Lam, ${ }^{4}$ and Hays and Baravilala, ${ }^{5}$ have found that while medical education reform in Asia is often based on a Western model, any changes have to take place in the context of local needs and culture. For instance, Lam and Lam noted that in Singapore's case, "because of an environment deeply entrenched in traditional education, [Yong Loo Lin School of Medicine] has decided not to fully adopt the [problem-based learning] curriculum."

Although Singapore has adopted the United Statesbased residency system, accredited by ACGME-I, we have also made modifications to take into account local circumstances, thus creating a unique Residency program. While improvements have already been made to the postgraduate medical education training, with residents benefitting from a more structured learning program and frequent evaluations, evolution of the residency system is still ongoing.

Singapore is the first country outside the United States to be conferred with accreditation by ACGME-I. To date, the only other countries outside of the United States to be so accredited are Qatar and United Arab Emirates. Our experience shows that overseas centers that want to implement the United States-style of residency will need sufficient resources to meet the accreditation standards, including sufficient faculty, resources for residents, such as electronic logging of duty hours and training hours, and physician extenders to help with the kind of repetitive service work that has only limited educational value. 
Acknowledgments: The authors wish to thank Ms Angeline Yong who was the program coordinator for pediatrics residency and helped to edit this article. Our thanks also go to the Residency faculty, Graduate Medical Education office and the KKH nursing administration for their strong support of the Residency program.

Funding/Support: None.

Other Disclosures: None.

Ethical Approval: Not applicable.

Disclaimer: None.

Previous Presentations: None.

\section{References}

1. Huggan PJ, Samarasekara DD, Archuleta $S$, Khoo SM, Sim JHJ, Sin CSP, Ooi SBS. The successful, rapid transition to a new model of graduate medical education in Singapore. Acad Med. 2012;87(9):122-127.

2. Yap ES, Goh W-P, Kee ACL, Chan YC. Implementation and experience of the first chief residency program in Singapore. J Grad Med Educ. 2010;2(3):346-348.

3. Singhealthresidency.com [Internet]. Pediatrics Residency Program. [cited 2015 November 30]. Singapore: Singapore Health Services Pte Ltd.; 2012. Available from http://www. singhealthresidency.com.sg/Documents/ Brochures/Pediatrics\%20LR.pdf.

4. Lam TP, Lam YYB. Medical education reform: The Asian experience. Acad Med. 2009;84(9):1313-1317.

5. Hays R, Baravilala W. Applying global standards across national boundaries: Lessons learned from an Asia-Pacific example. med Educ. 2004;38:582-586. 